\title{
Vitamin D Deficiency in Patients with Type 2 Diabetes Mellitus Leads to Vascular Complications
}

\author{
Salimah Navaz Gangji ${ }^{1}$ \\ University of Western Ontario
}

\begin{abstract}
The presence of hyperglycemia in individuals with Type 2 Diabetes Mellitus (T2DM) is associated with systemic complications within multiple organ systems. Specifically, patients with T2DM have an increased risk of developing vascular endothelial damage. Interestingly, patients with T2DM are often found to be deficient in vitamin D, a fat-soluble vitamin that not only plays a role in bone growth and gastrointestinal nutrient absorption, but insulin resistance as well. Thus, the purpose of this review is to summarize the literature that associates vitamin D deficiencies with vascular complications in both human and animal models with T2DM. This review will also summarize developments in genetic testing for VDR mutations and their potential role in diabetes progression, as well as the effects of vitamin D supplementation in patients with T2DM. Since T2DM is an increasingly prevalent disease, it is important to continue evaluating current research that investigates not only genetic causal factors for the disease, but also preventative options (such as vitamin D supplementation) that could potentially be used alongside pharmacological treatments.
\end{abstract}

\section{Introduction}

By the year 2030, the number of individuals with Type 2 Diabetes Mellitus (T2DM) is expected to increase from 371 million to over 500 million people worldwide (1). T2DM is characterized by the presence of hyperglycemia, where blood glucose levels remain elevated due to decreased insulin sensitivity (1). Uncontrolled hyperglycemia has been found to increase the mitochondrial production of reactive oxygen species (ROS) in the body $(2,3)$. ROS molecules are highly reactive and unstable oxygen-containing molecules that inappropriately interact with normal regulatory enzymes and proteins. Ultimately, this leads to the premature activation of many biochemical pathways that are thought to be responsible for the development of vascular complications in TD2M patients with fluctuating glucose levels (2). For example, studies show that microvascular dysfunction of the kidneys and eyes can be attributed to the hyperactivation of the protein-kinase $C$ (PKC) pathway which increases inflammatory cytokine release and growth factor gene expression $(2,3)$. Hyperactivation of the polyol pathway perpetuates microvascular endothelial damage due to the accumulation of sorbitol, while the accumulation of advanced glycylated end (AGE) products induces apoptosis and inflammation in retinal pericytes (3). Macrovascular dysfunction at the level of the vascular endothelium has been attributed to the inhibition of endothelial nitric-oxide synthase (eNOS) by ROS molecules, which decreases the normal vasodilation effect of $\mathrm{NO}$ and increases vascular resistance and inflammation (3).

Interestingly, T2DM patients with fluctuating glucose levels have been shown to have lower-thannormal levels of vitamin D (4). The optimal level of blood vitamin $\mathrm{D}$ to allow for normal bone growth and normal physiological function as set by Health Canada and the Institute of Medicine is any value equal to or greater than $50 \mathrm{nmol} / \mathrm{L}$ (5). Vitamin D deficiency refers to individuals with blood vitamin $D$ levels less than $30 \mathrm{nmol} / \mathrm{L}(5,6)$. A 2012 study found that nearly $32 \%$ of Canadians are below the 50 $\mathrm{nmol} / \mathrm{L}$ target, while $10 \%$ of all Canadians are vitamin D deficient (7). Large-scale longitudinal studies and smaller cohort studies conducted since 2010 have found that T2DM patients have lower vitamin $D$ levels than non-diabetic patients $(8,9,10)$. Additionally, the risk of developing T2DM in the first place is significantly higher in patients who are severely vitamin $\mathrm{D}$ deficient (less than $12 \mathrm{nmol} / \mathrm{L}$ ) compared to patients whose blood vitamin $D$ levels are well above $50 \mathrm{nmol} / \mathrm{L}$ (11).

Besides its involvement in skeletal growth and calcium regulation, the vitamin $\mathrm{D}$ receptor (VDR) is found on pancreatic beta cells and helps increase insulin sensitivity and secretion (12). Thus, deficiencies in vitamin D or the VDR are associated with the presence and progression of T2DM, where insulin sensitivity is impaired and leads to inflammation and further complications (13). In-vitro studies performed in mice have found that not only does vitamin $\mathrm{D}$ mediate the conversion of pro-insulin 
to its active form insulin, but that mice lacking functional VDRs on their pancreatic beta cells have decreased insulin secretion in response to glucose loading (11). More recent evidence shows that vitamin D supplementation may decrease fasting glucose levels, prevent pancreatic beta cell damage and increase insulin secretion in neonatal mice with STZ-induced diabetes (14).

This literature review will analyze recent studies that associate vitamin $D$ deficiency with vascular complications in patients with T2DM, and provide an overview of studies that attribute this complication to genetic defects in the VDR. This review will also summarize potential treatment options such as vitamin D supplementation and its implications for this subset of patients.

\section{Methods}

A systematic search was conducted between October 2016 through June 2017 using only Pubmed and Google Scholar databases. Combinations of various key words were used when searching the databases, such as: vitamin D deficiency, type 2 diabetes mellitus, vitamin D receptor polymorphism, cardiovascular complications, microvascular complications, vitamin D supplementation. Inclusion criteria included articles that were primary peer-reviewed articles from both basic and applied science disciplines. The only exclusion criteria were articles that were review papers or secondary articles, with the exception of particularly recent (since 2014) review articles whose findings were exceptionally concise and specific to the sub-topics discussed in this literature review. Articles published beyond the past 10 years were not included for the purpose of providing the most recent, up to date findings relevant to this topic, with the exception of one article published in 2006 that was one of the first cross-sectional studies to highlight the link between intimal medial thickening (IMT) and serum vitamin D levels in T2DM patients.

\section{Vitamin D deficiency may lead to macrovascular endothelial dysfunction in T2DM patients}

Macrovascular endothelial dysfunction is typically defined as any disturbances in larger arterial blood vessels that lead to complications such as myocardial infarctions and cerebrovascular incidents (15). It has been found that T2DM patients are more prone to developing cardiovascular disease due to the increased number of risk factors typically seen in these patients (hypertension, obesity), however changes in blood vessel structure have been observed in juvenile diabetic patients who do not have these risk factors $(16,17)$. While many factors contribute to macrovascular endothelial damage (presence of hypertension, dyslipidemia, genetic factors), the biochemical end-result is a decrease in the production of the vasodilator NO. This leads to a lack of plasminogen, an anti-clotting enzyme that is usually activated by NO (3). Ultimately, this causes increased inflammatory cell deposition in the endothelium and an increased number of fibrotic plaques in the larger blood vessels (3).

Recent studies indicate that vitamin $D$ may actually decrease the activity of these thrombotic pathways, thus decreasing inflammation and preventing atherosclerosis progression (18). Nardin and colleagues recently found that diabetic patients with vitamin $\mathrm{D}$ deficiency had increased prevalence of coronary artery disease (CAD) when examined using coronary angiograph (18). This finding was consistent for both male and female patients, although the researchers did not take into account the duration of the diabetes diagnosis for these patients.

Another study analyzed the IMT of the carotid artery in patients with T2DM, since IMT is a clinical marker for atherosclerosis (19). This study found that 130 diabetic patients with vitamin $D$ deficiency had significantly greater IMT compared to 260 diabetic patients who were not vitamin D deficient (19). In the data analysis, the researchers also excluded patients who were taking statin medications (drugs that decrease atherosclerosis plaque build-up) yet the results remained statistically significant. Thus, the researchers demonstrated that vitamin $\mathrm{D}$ deficiency has a strong association with increased IMT and atherosclerosis development.

Based on the results of these studies, it would be interesting to investigate any beneficial interactions between vitamin D supplementation and the drugs used to manage hyperglycemia in diabetic patients. For example, one of the drugs used to treat T2DM is a class called sulfonylureas. Unfortunately, sulfonylureas may increase the risk of cardiovascular complications hence why physicians preferentially prescribe metformin (20). It would therefore be of interest for researchers to investigate the cotreatment of sulfonylureas paired with vitamin $D$ supplements, and observe if this pairing can simultaneously treat T2DM while decreasing the risk of cardiovascular damage associated with the drug.

A 2013 study compared the effects of vitamin D supplementation on a large sample of T2DM patients taking various anti-hyperglycemia drugs 
such as insulin alone, insulin and oral medication, metformin alone, or sulfonylureas alone (21). They found that vitamin D supplementation did not adversely affect the anti-hyperglycemia effects of any of the drug treatment groups, and the insulin and oral medication combination group had the greatest improvement in blood HDL, triglycerides and blood pressure. Most interestingly, the sulfonylurea only group did not experience a significant change in blood HDL levels (21). This was an interesting finding because clinicians often observe a decrease in HDL in diabetic patients who are prescribed sulfonylureas (21). It can therefore be postulated that vitamin D supplementation may be an ideal solution to prevent the decrease in blood HDL levels typically seen in patients prescribed sulfonylureas.

\section{Microvascular complications in diabetic patients due to vitamin $D$ deficiency}

Microvascular complications are defined as damage that occurs in small blood vessels, leading to vascular dysfunction typically in the eyes (retinopathy) or nervous system (neuropathy) (15). For example, microvascular complications in diabetic patients are the leading cause of kidney failure (22). Research shows that this type of vascular damage occurs due to over-activation of the reninangiotensin-aldosterone system (RAAS), leading to abnormal protein excretion (proteinuria) (13). Recently, studies hypothesized that vitamin D may prevent RAAS hyperstimulation and vascular kidney damage. Usluogullari and colleagues analyzed over 500 diabetic patients over a three-month period. Not only did diabetic patients have increased prevalence of nephropathy, but those with vitamin $D$ deficiency had more severe nephropathy complications compared to diabetic patients who were not vitamin $D$ deficient (13).

The hypothesis that vitamin $D$ can prevent microvascular damage in patients with T2DM has also been recently tested on animal models. A study by Zhang and colleagues treated diabetic rats with calcitrol (the active form of vitamin D) and found that treatment decreased proteinuria in these rats and increased the expression of nephrin and podocin, proteins that are essential for supporting the structural integrity of kidney podocytes (23).

These studies collectively show that vitamin $D$ influences vascular integrity of the kidneys in both diabetic patients and animal models. Due to the severe morbidity and mortality associated with the progression of diabetic nephropathy, research needs to focus on prevention in addition to treatment. It would therefore be ideal for clinicians to screen newly diagnosed diabetic patients for vitamin $D$ deficiency and then immediately prescribe drug treatments to prevent microvascular complications before they happen.

\section{VDR genetic polymorphisms may explain vascular complications in diabetic patients}

Unfortunately, there have been variable results regarding the possibility that polymorphisms in the VDR gene influence T2DM progression. The inconsistent results are mostly likely due to the fact that the VDR gene is large $(75 \mathrm{~kb})$ and the fact that different ethnic groups appear to have varying allele frequencies thus making it difficult to isolate and study specific mutations in the gene (24).

A 2009 study genotyped patients with Type I diabetes, and found that the Bsml genotype of the VDR gene had a significant association with diabetic nephropathy (25). This finding was expected because Type I diabetes is a genetically inherited disease, but researchers wanted to further investigate genetic polymorphisms for T2DM, which is an adult-onset, non-genetic disease. To do so, researchers started looking at single nucleotide polymorphisms (SNPs) in the gene that encodes for the VDR, and they tried to categorize these SNPS depending on the ethnicity of the patients, as SNPs can vary depending on ethnicity. The results were conflicting: the Fokl polymorphism was strongly associated with T2DM in an Asian population, whereas another study found no association between Fokl and T2DM complications in a Polish Caucasian population (26).

Another recent study investigated changes in the SNPs for the Taql and Fokl polymorphisms of the VDR, specifically in post-menopausal women with T2DM. They found that there was no significant difference in the Taql or Fokl polymorphisms between diabetic post-menopausal women and the healthy control group (26). However, they found that the recessive ff phenotype in the Fokl polymorphism was associated with increased risk of CAD in these women (26). Unfortunately, this study had many limitations in that the sample size was small and while all the women were from Brazil, specific ethnic backgrounds were not taken into account during data analysis. Other researchers analyzed VDR mutations in 264 T2DM patients from the United Arab Emirates. They found that T2DM patients with the 
Taql mutation had higher LDL and total cholesterol levels compared to T2DM patients who had the Bsml mutation (27). They were even able to specify that the $A G$ and $G G$ genotypes within the Taql group had a stronger association with increased LDL and total cholesterol levels (27).

A more recent study wanted to observe the physiological changes associated with different genotype frequencies of the FokI VDR polymorphism in a group of T2DM patients with or without foot ulcers, a common end-result of diabetic peripheral neuropathy (28). The study compared serum levels of thiobarbituric acid reactive substances (TBARS), a marker of oxidative stress, and visually analyzed changes or development of foot ulcers in the patient population using Wagner's grading system for foot ulcers. The study found that the number of patients with TC and TT genotypes was significantly higher in the patient population with diabetic foot ulcers compared to the population without foot ulcers (28). In general, the patients with more severe disease progression in their foot ulcers were more likely to be carriers of the T allele in their VDR gene (28). Additionally, the frequency of the T allele was higher in patients with elevated levels of TBARS (28). Thus, the presence or absence of specific genotypes within the VDR gene in patients with T2DM may be predicative of a patient's risk for experiencing more aggressive vascular disease progression (28). Scientists should continue to collaborate and investigate which specific mutations in the VDR are predictive of vascular complications, and how dependent these mutations are on ethnicity.

\section{Use of vitamin D supplementation in preventing and treating vascular complications associated with T2DM}

One of the biggest controversies in this area of research is whether vitamin $D$ supplementation is actually effective for treating vascular damage in patients with T2DM. According to the 2016 chapter on Nutrition Therapy from Diabetes Canada Clinical Practice Guidelines, vitamin supplementation in general has yet to be recommended for diabetic patients, and vitamin D supplementation of $10 \mu \mathrm{g}$ (equivalent to $400 \mathrm{IU} / \mathrm{d}$ ) is only recommended for otherwise healthy patients who are 50 years of age and older (29). In terms of dosage, it should be noted that longitudinal and observational studies performed on diabetic patients typically administer larger doses (>1000 IU/d) compared to the $400 \mathrm{IU} / \mathrm{d}$ daily supplementation recommended by Health Canada. This is because doses $>1000 \mathrm{IU} / \mathrm{d}$ are thought to be the optimal dose required to cause detectable, sustainable increases in serum vitamin D levels, and larger doses are usually administered for shorter periods of time $(30,31)$.

A recent study administered 50000 IU of a vitamin $D$ supplement every two weeks for six months to T2DM patients with CAD. Supplementation improved their glycemic control and blood NO levels compared to T2DM patients who received a placebo (32). Another study found that a single, $100000 \mathrm{IU}$ dose of vitamin $\mathrm{D}$ given to patients with T2DM improved endothelial function (vasodilation of the brachial artery) but only significantly decreased systolic blood pressure compared to patients who received a placebo (33). Conversely, one study found that T2DM patients given $4000 \mathrm{IU} /$ day for two months had slightly improved $\mathrm{HbA} 1 \mathrm{c}$ levels compared to T2DM patients who did not receive supplementation, but there was no improvement in relative insulin resistance (34). Additionally, a study using diabetic rats investigated the presence of serum asymmetric dimethylarginine (ADMA), a marker of vascular damage that decreases nitric oxide production and thus decreases arterial elasticity (35). They found that the integrity of the aorta was significantly improved in diabetic rats that received $500 \mathrm{IU} / \mathrm{kg}$ of vitamin $\mathrm{D}$ supplementation for ten weeks compared to untreated diabetic rats (35). It is therefore important that future studies determine a specific supplementation dose range that is effective in the majority of T2DM patients regardless of any other pre-existing or progressing diseases that they may already have, such as CAD. It should be noted that vitamin $D$ toxicity caused by excessive supplementation is actually uncommon, and only occurs when blood vitamin D levels exceed 250-375 $\mathrm{nmol} / \mathrm{L}$ (36).

\section{Conclusion}

It is evident that patients with T2DM are more likely to suffer from vitamin D deficiency, and this deficiency can lead to many vascular complications. Macrovascular (at the level of the arteries) and microvascular (at the level of blood vessels and individual organs) complications are equally prominent in T2DM. Studies hypothesize that genetic polymorphisms in the vitamin $D$ receptor may be responsible for the vascular complications seen in T2DM patients. However, the results of these studies are conflicting, as are the results of studies investigating vitamin D supplementation as an exploratory treatment to prevent vascular damage in 
this subset of patients. Genetic predisposition and vitamin $D$ supplementation should continue to be investigated with regards to patients with T2DM, as the implications are important for this subset of patients. Hopefully, physicians would have clearer guidelines for scanning newly diagnosed T2DM patients for genetic defects in VDR. Early detection of such mutations would allow physicians to proactively prescribe other drugs to prevent vascular damage in these patients. Additionally, as more longitudinal studies continue to investigate the effects of vitamin D supplementation on vascular damage and glycemic control, physicians will hopefully have a greater understanding of whether long term supplementation is actually effective and what exact dosages of vitamin $D$ are required to prevent and treat vascular damage in this subset of patients.

\section{References}

1. Priyadarsini S, McKay TB, Sarker-Nag A, Allegood J, Chalfant C, Ma J-XX, et al (2016). Complete metabolome and lipidome analysis reveals novel biomarkers in the human diabetic corneal stroma. Exp Eye Res. 153:90-100

2. Asmat U, Abad K, \& Ismail K. (2016). Diabetes mellitus and oxidative stress-A concise review. Saudi Pharm J, 24(5), 547553.

3. Cade WT. (2008). Diabetes-related microvascular and macrovascular diseases in the physical therapy setting. Physical therapy, 88(11), 1322-35.

4. Kostoglou-Athanassiou I, Athanassiou P, Gkountouvas A, Kaldrymides P. Vitamin D and glycemic control in diabetes mellitus type 2. Ther Adv Endocrinol Metab. 2013 Aug 4;4(4):122-8.

5. Aghajafari F, Field CJ, Kaplan BJ, Rabi DM, Maggiore JA, O'Beirne $\mathrm{M}$ et al. (2016). The current recommended vitamin D intake guideline for diet and supplements during pregnancy is not adequate to achieve vitamin D sufficiency for most pregnant women. PLoS ONE, 11(7).

6. Kennel KA, Drake MT \& Hurley DL. (2010). Vitamin D deficiency in adults: when to test and how to treat. Mayo Clinic Proceedings, 85(8), 752-758.

7. Roberts K, Shields M, Groh M, et al. Overweight and obesity in children and adolescents: Results from the 2009 to 2011 Canadian Health Measures Survey. Health Reports. September 2012;23(3):3-6.

8. Gagnon C, Lu ZX, Magliano DJ, Dunstan DW, Shaw JE, Zimmet PZ et al. (2011). Serum 25-Hydroxyvitamin D, calcium intake, and risk of type 2 diabetes after 5 years: results from a national, population-based prospective study (the Australian Diabetes, Obesity and Lifestyle study). Diabetes Care, 34(5), 1133-1138.

9. Taheri E, Saedisomeolia A, Djalali M, Qorbani M, \& Madani Civi M. (2012). The relationship between serum 25-hydroxy vitamin $\mathrm{D}$ concentration and obesity in type 2 diabetic patients and healthy subjects. J Diabetes Metab Disord, 11, 16.

10. Bayani MA, Akbari R, Banasaz B \& Saeedi, F. (2014). Status of vitamin-D in diabetic patients. Caspian J Intern Med, 5(1), 40-42.

11. Grammatiki M, Rapti E, Karras S, Ajjan RA \& Kotsa, K. (2017). Vitamin $D$ and diabetes mellitus: Causal or casual association? Rev Endocr Metab Disord.
12. Talaei A, Mohamadi M, Adgi Z. The effect of vitamin D on insulin resistance in patients with type 2 diabetes. Diabetol Metab Syndr. 2013 Feb 2;5(1):8.

13. Usluogullari CA, Balkan F, Caner S, Ucler R, Kaya C, Ersoy $\mathrm{R}$, et al. The relationship between microvascular complications and vitamin $D$ deficiency in type 2 diabetes mellitus. BMC Endocr Disord. 2015 Jun 4;15:33.

14. Wang G, Hu C, Hu C, Ruan L, Bo Q \& Li L. (2013). [Impact of oral vitamin $\mathrm{D}$ supplementation in early life on diabetic mice induced by streptozotocin]. Journal of hygiene research, 42(3), 455-9.

15. Alaboud AF, Tourkmani AM, Alharbi TJ, Alobikan $A H$, Abdelhay O, Al Batal SM, et al. Microvascular and macrovascular complications of type 2 diabetic mellitus in Central, Kingdom of Saudi Arabia. Saudi Med J. 2016 Dec 4;37(12):1408-11.

16. Dayem SM, Battah AA, El Bohy AE. Assessment of Increase in Aortic and Carotid Intimal Medial Thickness in Type 1 Diabetic Patients. Open Access Maced J Med Sci. 2016 Dec 4;4(4):630-5.

17. Martín-Timón I, Sevillano-Collantes C, Segura-Galindo A \& del Cañizo-Gómez FJ. (2014). Type 2 diabetes and cardiovascular disease: Have all risk factors the same strength? World $\mathrm{J}$ Diabetes, 5(4), 444-470

18. Nardin M, Verdoia M, Schaffer A, Barbieri L, Marino P, De Luca G. Vitamin D status, diabetes mellitus and coronary artery disease in patients undergoing coronary angiography. Atherosclerosis. 2016 Jul 5;250:114-21.

19. Targher G, Bertolini L, Padovani R, Zenari L, Scala L, Cigolini $M$, et al. Serum 25-hydroxyvitamin D3 concentrations and carotid artery intima-media thickness among type 2 diabetic patients. Clin Endocrinol (Oxf). 2006 Nov 3;65(5):593-7.

20. Ou S-MM, Shih C-JJ, Chao P-WW, Chu H, Kuo S-CC, Lee Y$\mathrm{JJ}$, et al. Effects on Clinical Outcomes of Adding Dipeptidyl Peptidase-4 Inhibitors Versus Sulfonylureas to Metformin Therapy in Patients With Type 2 Diabetes Mellitus. Ann Intern Med. 2015 Nov 2;163(9):663-72.

21. Alkharfy KM, Al-Daghri NM, Sabico SB, Al-Othman A Moharram O, Alokail MS, Al-Saleh Y et al. (2013). Vitamin D supplementation in patients with diabetes mellitus type 2 on different therapeutic regimens: a one-year prospective study. Cardiovasc diabetol, 12, 113

22. Chen S, Villalta SA, Agrawal DK. FOXO1 Mediates Vitamin D Deficiency-Induced Insulin Resistance in Skeletal Muscle. J Bone Miner Res. 2016 Mar 2;31(3):585-95.

23. Zhang X-LL, Guo Y-FF, Song Z-XX, Zhou M. Vitamin D prevents podocyte injury via regulation of macrophage M1/M2 phenotype in diabetic nephropathy rats. Endocrinology. 2014 Dec 1;155(12):4939-50.

24. Mahjoubi I, Kallel A, Sbaï MH, Ftouhi B, Ben Halima M, Jemaa $Z$, et al. Lack of association between Fokl polymorphism in vitamin $\mathrm{D}$ receptor gene (VDR) \& type 2 diabetes mellitus in the Tunisian population. Indian J Med Res. 2016 Jul 5;144(1):4651.

25. Bućan K, Ivanisević M, Zemunik T, Boraska V, Skrabić V, Vatavuk Z, et al. Retinopathy and nephropathy in type 1 diabetic patients--association with polymorphysms of vitamin D-receptor, TNF, Neuro-D and IL-1 receptor 1 genes. Coll Antropol. 2009 Dec 2;33 Suppl 2:99-105.

26. Maia J, da Silva AS, do Carmo RF, de Mendonça TF, Griz LH, Moura $P$, et al. The association between vitamin $D$ receptor gene polymorphisms (Taql and Fokl), Type 2 diabetes, and micro-/macrovascular complications in postmenopausal women. Appl Clin Genet. 2016 Jan 5;9:131-6.

27. Safar HA, Chehadeh SE, Abdel-Wareth L, Haq A, Jelinek HF, EIGhazali G \& Anouti FA (2017). Vitamin D receptor gene 
polymorphisms among Emirati patients with type 2 diabetes mellitus. J. Steroid Biochem. Mol. Biol.

28. Soroush N, Radfar M, Hamidi AK, Abdollahi M, Qorbani M, Razi F, Esfahani EN et al. (2017). Vitamin D receptor gene Fokl variant in diabetic foot ulcer and its relation with oxidative stress. Gene, 599, 87-91.

29. Harper W, Clement M, Goldenberg R, et al. Canadian diabetes association 2013 clinical practice guidelines for the prevention and management of diabetes in canada: pharmacologic management of type 2 diabetes. Can J Diabetes 2013;37(suppl 1):S61-S68.

30. Penckofer S, Kouba J, Wallis DE, \& Emanuele MA. (2008). Vitamin $D$ and diabetes let the sunshine in. Diabetes Educ, 34(6), 939-passim.

31. Mager DR, Jackson ST, Hoffmann MR, Jindal K \& Senior PA. (2014). Vitamin D supplementation and bone health in adults with diabetic nephropathy: the protocol for a randomized controlled trial. BMC endocrine disorders, 14, 66.

32. Farrokhian A, Raygan F, Bahmani F, Talari HR, Esfandiari R, Esmaillzadeh A. \& Asemi Z. (2017). Long-term vitamin D supplementation affects metabolic status in vitamin D-deficient type 2 diabetic patients with coronary artery disease. J. Nutr.147(3), 384-389.

33. Sugden JA, Davies JI, Witham MD, Morris AD, Struthers AD. Vitamin D improves endothelial function in patients with Type 2 diabetes mellitus and low vitamin D levels. Diabetic Med. wiley; 2008;25(3):320-5.

34. Yousefi Rad E, Djalali M, Koohdani F, Saboor-Yaraghi AA, Eshraghian MR, Javanbakht MH, Saboori S et al. (2014). The effects of vitamin D supplementation on glucose control and insulin resistance in patients with diabetes type 2: a randomized clinical trial study. Iran J Public Health, 43(12), 1651-6.
35. Salum E, Kampus $P$, Zilmer M, Eha J, Butlin M, Avolio AP, et al. Effect of vitamin $D$ on aortic remodeling in streptozotocininduced diabetes. Cardiovasc Diabetol. 2012 Jul 5;11:58.

36. Baz-Hecht M \& Goldfine AB. (2010). The impact of vitamin D deficiency on diabetes and cardiovascular risk. Curr Opin Endocrinol Diabetes Obes, 17(2), 113-9. 